\title{
Gas chromatography-mass spectrometric method-based urine metabolomic profile of rats with pelvic inflammatory disease
}

\author{
WEI ZOU ${ }^{1}$, XIAOKE WEN $^{1}$, XIAOQI SHENG $^{2}$, YI ZHENG $^{1}$, ZUOQI XIAO $^{1}$, JIEYING LUO ${ }^{3}$, \\ SHUQIONG CHEN ${ }^{4}$, YICHAO WANG ${ }^{2}$, ZENENG CHENG $^{5}$, DAXIONG XIANG $^{6}$ and YICHU NIE $^{7}$ \\ ${ }^{1}$ Key Laboratory of Hunan Province for Traditional Chinese Medicine in Obstetrics and Gynecology Research; \\ ${ }^{2}$ Technical Research Centre of Clinical Preventive and Treatment for Children, \\ The Maternal and Child Health Hospital of Hunan Province, Changsha, Hunan 410008; \\ ${ }^{3}$ College of Pharmacy, Hunan University of Traditional Chinese Medicine, Changsha, Hunan 410007; \\ ${ }^{4}$ Traditional Chinese Medicine Department of Gynecology, The Maternal and Child Health Hospital of Hunan Province, \\ Changsha, Hunan 410008; ${ }^{5}$ School of Pharmaceutical Sciences, Central South University; \\ ${ }^{6}$ Clinic Pharmacy Research Laboratory, Second Xiangya Hospital of Central South University, Changsha, Hunan 410011; \\ ${ }^{7}$ State Key Laboratory of Respiratory Diseases, The First Affiliated Hospital of Guangzhou Medical University, \\ Guangzhou, Guangdong 510120, P.R. China
}

Received February 8, 2015; Accepted February 23, 2016

DOI: $10.3892 /$ etm.2016.3142

\begin{abstract}
Pelvic inflammatory disease (PID) can lead to a poor outcome of severe sequelae, and the current methods of clinical diagnosis are not satisfactory. Metabolomics is an effective method for the identification of disease-related metabolite biomarkers to facilitate disease diagnosis. However, to the best of our knowledge, no PID-associated metabolomic study has yet been carried out. The metabolomic changes of rats with PID were investigated in the present study. A PID model was constructed by the multi-pathogenic infection of
\end{abstract}

Correspondence to: Mr. Xiaoke Wen, Key Laboratory of Hunan Province for Traditional Chinese Medicine in Obstetrics and Gynecology Research, The Maternal and Child Health Hospital of Hunan Province, 53 Xiangchun Road, Changsha, Hunan 410008, P.R. China

E-mail: wxk19631214@sina.com

Dr Yichu Nie, State Key Laboratory of Respiratory Diseases, The First Affiliated Hospital of Guangzhou Medical University, 151 Yanjiang Road, Guangzhou, Guangdong 510120, P.R. China E-mail: nieyichu2@126.com

Abbreviations: GC/MS, gas chromatography-mass spectrometry; PID, pelvic inflammatory disease; PCA, principal component analysis; PLS-DS, partial least squares-discriminant analysis; TCA cycle, citric acid cycle; BSTFA-TMCS, N,O-bis(trimethylsilyl)-trifl uoroacetamide-trimethylchlorosilane; CRP, C-reactive protein; IL, interleukin; QC, quality control; VIP, variable importance in the projections; TLR, toll-like receptor; LTB4, leukotriene B4; PGE2, prostaglandin E2

Key words: pelvic inflammatory disease, metabolomic, biomarker, GC-MS, urine, rats the upper genital tract in rats. Infiltration of inflammatory cells and elevated expression levels of the cytokines interleukin (IL)-1 $\beta$ and IL-6 in the uterus and fallopian tubes validated the disease model. Gas chromatography-mass spectrometry coupled with derivatization was used to determine the urine metabolomic profile. Principal component analysis and partial least squares-discriminant analysis of the data sets showed a clear separation of metabolic profiles between rats with PID and control rats. Eighteen differentiating metabolites were found, including four amino acids, three fatty acids, nine organic acids, and two sugars, which indicated alterations in sugar metabolism, the citric acid cycle, amino acid metabolism and fatty acid metabolism. These metabolites could be potential biomarkers of PID, and this research may offer a new approach to evaluate the effect of anti-PID drugs in pre-clinical or clinical trials.

\section{Introduction}

Pelvic inflammatory disease (PID) is a common disorder induced by ascending infection of the upper female genital tract by, for example, Neisseria gonorrhoeae, Chlamydia trachomatis, genital mycoplasmas, gram-negative bacteria or gram-positive bacteria (1-4), the symptoms of which include endometritis, salpingitis and peritonitis (5). This disease is considered to be a great threaten to women as it can lead to a poor outcome with severe sequelae, such as tubal factor infertility, ectopic pregnancy and chronic pelvic pain. The diagnosis of PID is dependent upon evaluation of the patient's history, physical examination, laboratory studies and imaging (5). However, the sensitivity and specificity of clinical diagnosis have been reported to be only 87 and $50 \%$, respectively (6). Although laparoscopy is the gold standard for diagnosis, its routine use is limited by poor compliance (7). Therefore, a novel and non-invasive method is necessary for clinical diagnosis of PID. 
Metabolomics, a key technology for the evaluation of system biology, focuses on the comprehensive qualification and quantification of all endogenous metabolites of low molecular mass in vivo, and thus reveals the pathological and physiological changes of the whole biosystem from the aspect of metabolism $(8,9)$. Metabolomics has been carried out to identify disease-related metabolites as biomarkers that contribute to the non-invasive diagnosis of diseases. A previous study has found that 3-hydroxybutyrate, $\mathrm{N}$-acetyl-glycine, 3-hydroxy-2-methyl-butanoic acid and nonanedioic acid in the plasma can serve as biomarkers for the diagnosis of breast cancer (10). The levels of sarcosine in urine can contribute to the diagnosis of prostate cancer (11), and desmosterol in plasma has been identified as a new specific biomarker of Alzheimer's disease (12). However, to the best of our knowledge, no metabolomic study has yet been carried out to search for novel metabolite biomarkers of PID.

Gas chromatography coupled with mass spectrometry (GC/MS) is one of the core analytical methods for metabolomic study and has been used as a platform in non-targeted metabolomic analysis $(13,14)$. Through GC-MS analysis, the chemical structure of metabolites can be inferred by comparing the mass spectrometric fragmentation pattern with mass spectral libraries, which facilitates the identification of biomarkers. Silylation is a common method of derivatization, the application of which in GC-MS analysis can enable the detection of more metabolites, including carbohydrates, amino acids and nucleosides (15).

Following renal concentration, urine amplifies the circulating levels of metabolites and thus exhibits metabolomic alterations more clearly. In addition, urine collection is non-invasive. Hence, urine is of interest in metabolomic research. In the majority of medical research, animal experiments are usually carried out first to estimate the importance of conducting clinical experiments and offer valuable information. Therefore, on the basis of silylation and GC-MS analysis, the present urine non-targeted metabolomic study was carried out in a rat model of PID to illustrate the metabolic alterations associated with PID, and identify the potential biomarkers of PID accordingly.

\section{Materials and methods}

Reagents and materials. Ultrapure water was produced using a Milli-Q Plus Water Purification System (EMD Millipore, Milford, MA, USA). Pentobarbital was purchased from Xiya Reagent Co., Ltd. (Chengdu, China). Progesterone injection was obtained from Zhejiang Xianju Pharmaceutical Co., Ltd. (Taizhou, China). N,O-Bis(trimethylsilyl)-trifluoro-acetamid e-trimethylchlorosilane (BSTFA-TMCS) (99:1, v/v), urease, margaric acid, tropic acid, methoxylamine hydrochloride, pyridine and metabolite standards were purchased from Sigma-Aldrich (St. Louis, MO, USA). Absorbable gelatin sponge was purchased from Jinling Pharmaceutical Co., Ltd. (Nanjing, China).

PID model construction and sample collection. The experimental procedures were approved by the Animal Care and Use Committee of Central South University (Changsha,
China). The harm to animals was minimized with the greatest efforts. Sixteen female specific pathogen-free Sprague Dawley (SD) rats, aged 9 weeks and weighing 210-230 g, were purchased from Silaikejingda Experimental Animal Co., Ltd. (Changsha, China). They were randomly divided into two groups, namely the control group and the PID group. Standard laboratory chow was available ad libitum through the whole experiment. All the rats were acclimated for a week and then injected subcutaneously with $10 \mathrm{mg}$ progesterone 7 days prior to experimentation. PID modeling was conducted with reference to a previous method $(16,17)$. Ureaplasma urealyticum (t-strain mycoplasma) and a pathogenic Escherichia coli strain (No. 0573) were obtained from Clinical Laboratory Department of the Maternal and Child Health Hospital of Hunan Province (Changsha, China). An absorbable gelatin sponge, with a volume of $0.125 \mathrm{ml}$, was immerged into a microbe-mixing solution with a $U$. urealyticum concentration of $1 \times 10^{8} \mathrm{ccu} / \mathrm{ml}$ and E. coli concentration of $1 \times 10^{8} \mathrm{cfu} / \mathrm{ml}$. Then, the cervix of each rat in the PID group was implanted with the microbe-containing gelatin sponge, and the rat was positioned upside down for $3 \mathrm{~min}$. The cervixes of control group rats were implanted with gelatin sponges immerged in saline. This infection procedure was conducted once every 2 days and repeated four times. Surface disinfection was carried out with $70 \%$ alcohol following each infection. On the 21st day from the first infection, a $24 \mathrm{~h}$ urine sample of each rat was collected and stored at $-80^{\circ} \mathrm{C}$. Then, vaginal swab samples from rats were obtained to detect $U$. urealyticum, and $E$. coli using a mycoplasma detection kit and TDR-300B automatic microbial analysis system (Tiandiren Biotech Co., Ltd., Changsha, China), respectively. After that, rats were anesthetized with pentobarbital (dose, $30 \mathrm{mg} / \mathrm{kg}$ ). Plasma and the right uterus and fallopian tube were collected and stored at $-80^{\circ} \mathrm{C}$, and the left uterus and fallopian tube were used for histological examination. Then, all the rats were sacrificed via cervical dislocation.

Histological examination. The left uterus and fallopian tube of each rat were fixed in neutral-buffered formalin (10\%), embedded in paraffin, cut into $2-\mu \mathrm{m}$ sections, and stained with hematoxylin and eosin (H\&E). Three different fields for each tissue sample were examined using light microscopy (DM5001; Leica Microsystems GmbH, Wetzlar, Germany) at a magnification of $x 100$ by a blinded observer. The inflammation of the uterus and fallopian tube of each animal was semi-scored by the observer's evaluation of the degree of inflammatory cell infiltration (graded from 0 to 3 ) for each of the three fields.

$C$-reactive protein (CRP), interleukin (IL)-1 $\beta$ and IL-6 determinations. The concentration of CRP in plasma was measured using enzyme-linked immunosorbent assay (ELISA) kits (RayBiotech, Norcross, GA, USA). The right upper genital tract (including uterus and fallopian tube) of each rat was weighed, followed by the addition of physiological saline at the ratio of 5:1 (v/w) and homogenization. The total protein of the homogenate was measured using a bicinchoninic acid assay kit (Beyotime Institute of Biotechnology, Shanghai, China). The concentrations of IL- $1 \beta$ and IL-6 
in the homogenate were determined using the ELISA kits and presented in units of $\mu \mathrm{g} / \mathrm{g}$ protein of homogenate. These procedures were performed according to the protocols of the manufacturers.

Urine sample preparation. Thawed urine samples were diluted to give a creatinine concentration of $2.5 \mathrm{mmol} / \mathrm{l}$ as determined using a JEOL JCA-BM1650 clinical biochemistry analyzer (JEOL Ltd., Tokyo, Japan). A 30-unit quantity of urease was added to $100 \mu \mathrm{l}$ urine and the mixture was incubated for $30 \mathrm{~min}$ at $37^{\circ} \mathrm{C}$. Then, $50 \mu \mathrm{l}$ of a mixed solution of margaric acid and tropic acid, each of concentration $0.5 \mathrm{mg} / \mathrm{ml}$, was added as an internal standard. An aliquot of $800 \mu \mathrm{l}$ ethanol was mixed with the sample, and then the mixture was centrifuged at $12,000 \mathrm{x}$ g for $15 \mathrm{~min}$. The supernatant was transferred to a new tube and stored at $-20^{\circ} \mathrm{C}$ for $10 \mathrm{~min}$. After drying the supernatant in a vacuum dryer at room temperature, $100 \mu \mathrm{l}$ methoxylamine hydrochloride (20 $\mathrm{mg} / \mathrm{ml}$ in pyridine) was added with mixing, and the resultant mixture was incubated at $37^{\circ} \mathrm{C}$ for $2 \mathrm{~h}$. Then, $100 \mu \mathrm{l}$ BSTFA-TMCS was added and the mixture was heated to $70^{\circ} \mathrm{C}$ for $1 \mathrm{~h}$. A quality control (QC) sample was prepared by mixing urine from each rat and handling according to the above protocol.

GC-MS analysis. GC-MS analysis was undertaken on an Agilent 7890 gas chromatography system equipped with an Agilent 5975C mass analyzer (Agilent Technologies, Inc., Palo Alto, CA, USA). Separation was conducted on an Agilent DB-5MS capillary column (30 m x $0.25 \mathrm{~mm}$ internal diameter $\mathrm{x} 0.25 \mu \mathrm{m}$ film thickness). Each $1 \mu \mathrm{l}$ aliquot of the derivatized solution or reference standard was injected in the splitless mode and helium was used as the carrier gas with flow rate of $1.5 \mathrm{ml} / \mathrm{min}$. The temperatures of the inlet, transfer line and ion source were maintained at 250, 300 and $230^{\circ} \mathrm{C}$, respectively. The $\mathrm{GC}$ temperature programming was set to $4 \mathrm{~min}$ isothermal heating at $60^{\circ} \mathrm{C}$, followed by the first ramp at $6^{\circ} \mathrm{C} / \mathrm{min}$ to $150^{\circ} \mathrm{C}$ and holding for $8 \mathrm{~min}$, second ramp at $8^{\circ} \mathrm{C} / \mathrm{min}$ to $280^{\circ} \mathrm{C}$ and holding for $10 \mathrm{~min}$, and third ramp at $12^{\circ} \mathrm{C} / \mathrm{min}$ to $320^{\circ} \mathrm{C}$. Data acquisition was achieved using $\mathrm{MS}$ in the electron impact mode at $70 \mathrm{eV}$ and in the full-scan monitoring mode with a mass to charge $(\mathrm{m} / \mathrm{z})$ ratio from 35 to 750 . The chromatogram acquisition and detection of mass spectral peaks were performed using G1701EA GC/MD ChemStation software (Agilent Technologies, Inc.).

Data analysis and potential biomarker identification. The peak areas of metabolites in each sample were normalized to the peak area of creatinine. Internal standards were used to calibrate the retention time of all metabolites and monitor sampling and instrumental condition. Then, a data set of all samples, consisting of the retention time and the normalized peak area of metabolites, was generated and imported into SIMCA-P software (version 11.5; Umetrics; MKS Instruments, Umeå, Sweden) for multivariate analysis. The data were processed by unit variance scaling and were mean-centered, followed by multivariate analysis including principal component analysis (PCA) and partial least squares discriminant analysis (PLS-DA) between the PID group and control group. The unsupervised statistical analysis
PCA was used to describe associations and patterns among a set of variables; R2X and Q2 are two measures of PCA model quality. Metabolites relevant for group discrimination were selected by the values of variable importance in the projections (VIP) $>1$ constructed from PLS-DA analysis. The Student's t-test was employed for the metabolites with VIP $>1$, and the differentiating metabolites were selected when $\mathrm{P}<0.05$ with the Benjamini and Hochberg procedure to control the false discovery rate (FDR) (18). The mass fragmentation patterns of these potential biomarkers were compared with the NIST/EPA/NIH Mass Spectral Library 2011 (version NIST11; Gaithersburg, MD, USA) to predict their chemical structures. The further identification of these differentiating metabolites was performed by comparing their mass spectra and chromatographic retention times to those of standards.

Statistical analysis. Data are presented as mean \pm standard deviation (SD). Statistical analysis was performed by unpaired Student's t-test using SPSS for Windows 16.0 (SPSS, Inc., Chicago, IL, USA). P $<0.05$ was considered to indicate a statistically significant result.

\section{Results}

PID model construction. The vaginal swab samples of rats from the PID group tested positive for both $U$. urealyticum and $E$. coli, whereas the control rats tested negative for the two pathogens. These results indicated that infection in the PID group rats was successfully achieved. Histological examination revealed that, in comparison with control rats, upper genital tract infection with these pathogens caused significant inflammation, of the uterus and the fallopian tubes (Fig. 1A-D), including mass neutrophil and lymphocyte infiltration. Semi-quantitative scoring of the inflammation of the uterus (Fig. 1E) and fallopian tubes (Fig. 1F) showed a significant difference between the PID and control groups. The CRP levels in plasma, and expression levels of IL-1 $\beta$ and IL-6 in the upper genital tract (including the uterus and fallopian tube) were significantly higher in PID model rats, as compared with the control animals (Fig. 2), which indicated that an inflammatory response occurred in upper genital tract.

Assessment of the repeatability and stability of the analytical method. A representative chromatogram for the GC-MS coupled with derivatization method is shown in Fig. 3. Repeatability and stability of the analytical method are essential for obtaining valid metabolomic data. In the present study, a stability test was performed by analyzing 6 injections of a QC sample, and the relative standard deviations (RSDs) of eight common ions were $<0.27 \%$ for retention times and $<5.35 \%$ for peak areas. The repeatability was evaluated by the analysis of 6 QC samples, with the RSDs of peak area $<11.28 \%$. These results validated the analytical method for metabolite determination.

Multivariate statistical analysis. A three-component PCA model was selected to reveal the general metabolic differences between groups, with $\mathrm{R} 2 \mathrm{X}=0.751$ and $\mathrm{Q} 2=0.412$. As presented 

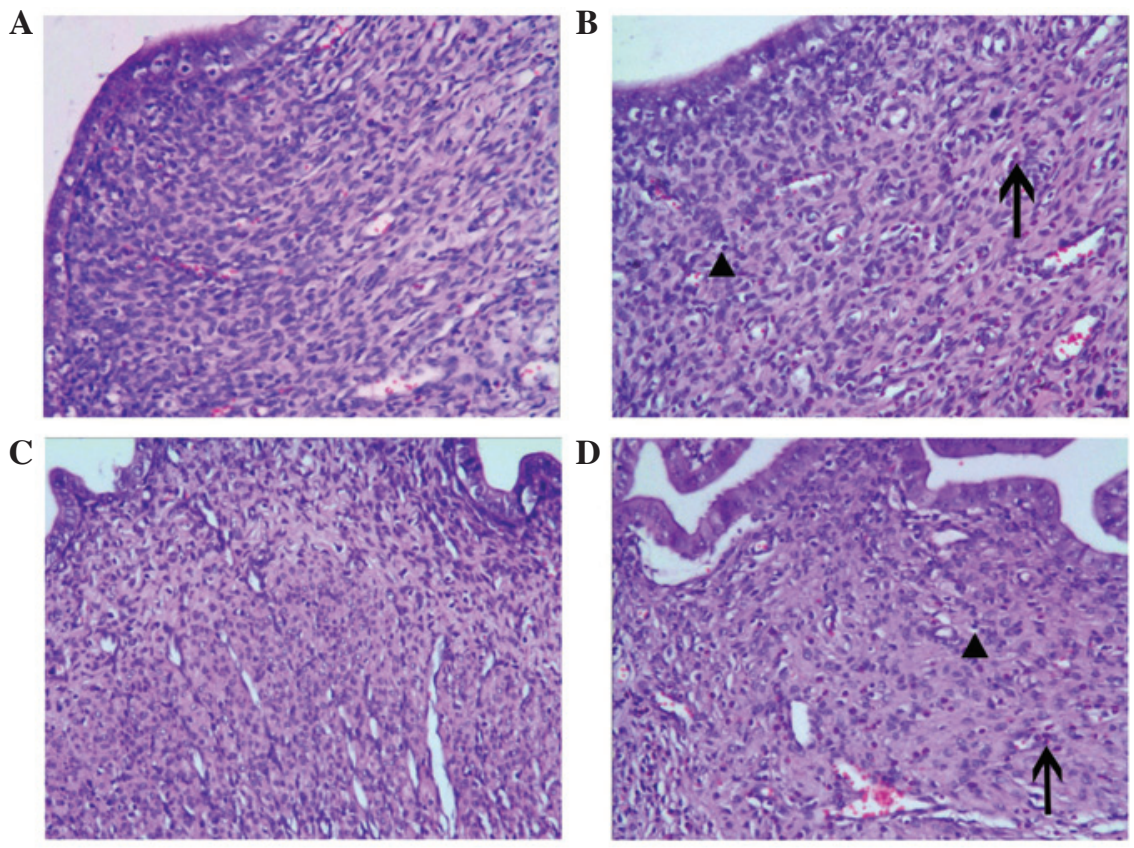

$\mathbf{E}$

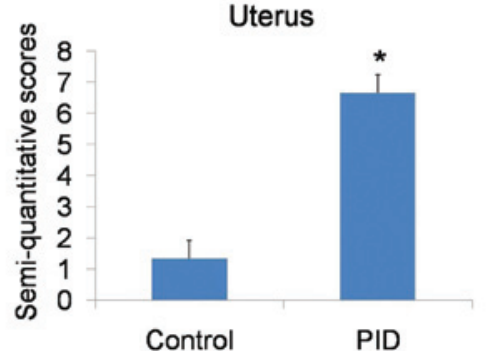

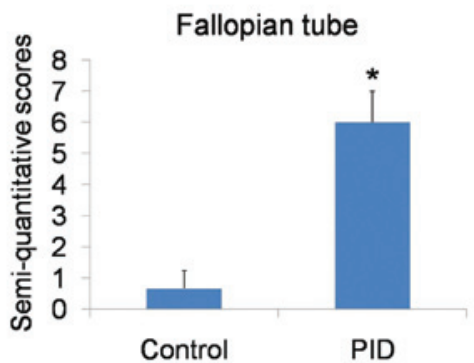

Figure 1. Histological changes of the uterus and fallopian tube after pathogenic infection. Representative micrographs of uterus and fallopian tube stained with hematoxylin and eosin (magnification, x100) are shown. Uterus of (A) a control rat and (B) a PID rat, and fallopian tube of (C) a control rat and (D) a PID rat. Lymphocyte cell infiltration is indicated with $\boldsymbol{\Lambda}$, and positive staining of neutrophils is indicated with $\uparrow$. Histological semi-quantitative scores of inflammatory cell infiltration in the (E) uterus and (F) fallopian tube are presented. Each bar represents the mean \pm standard deviation. "P<0.01 vs. the control group, $\mathrm{n}=3$ ). PID, pelvic inflammatory disease.
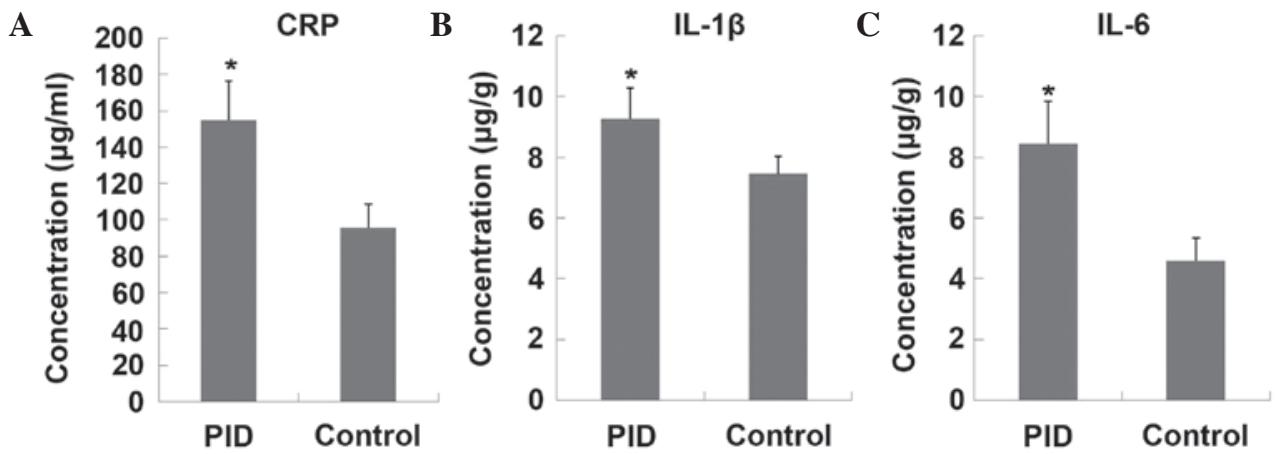

Figure 2. Expression changes of (A) CRP in the plasma and (B) IL-1 $\beta$ and (C) IL-6 in the upper genital tract (including uterus and fallopian tube) following pathogenic infection. Each bar represents the mean \pm standard deviation. ${ }^{*} \mathrm{P}<0.01$ vs. the control group, $\mathrm{n}=8$ ). CRP, C-reactive protein; IL, interleukin; PID, pelvic inflammatory disease.

in Fig. 4A, the PID group was clearly separated from the control group. Subsequently, a PLS-DA was employed to further elucidate the metabolic differentiation associated with PID. A clear separation is shown in Fig. 4B, with three predictive components and three orthogonal components ( $\mathrm{R} 2 \mathrm{X}=0.652, \mathrm{R} 2 \mathrm{Y}=0.934, \mathrm{Q} 2=0.720$ ). These results indicated that the metabolic profile of rats was evidently disturbed by PID.
Potential biomarker identification. Eighteen differentiating metabolites were selected as potential biomarkers with VIP $>1$ and $\mathrm{P}<0.05$, followed by the identification of chemical structures using commercial standards. Typical mass spectra of phenylalanine in urine and phenylalanine standard are presented in Fig. 5. A heat map of the potential biomarkers is presented in Fig. 6, which visualizes the fold level changes between the rats in the PID and control groups. These differentiating metabolites 


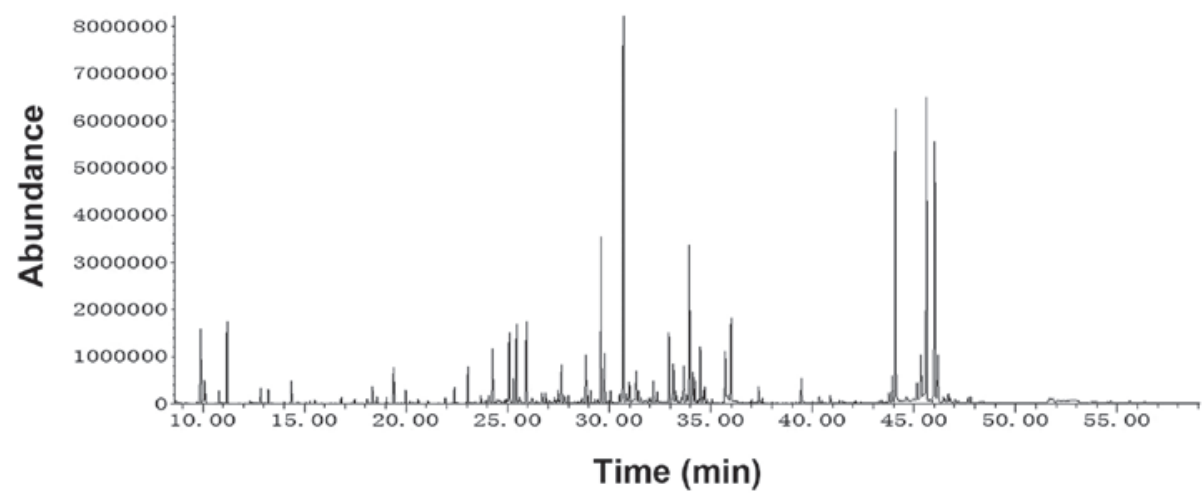

Figure 3. Representative base peak intensity chromatogram of rat urine obtained by gas chromatography-mass spectrometry coupled with a derivatization method.
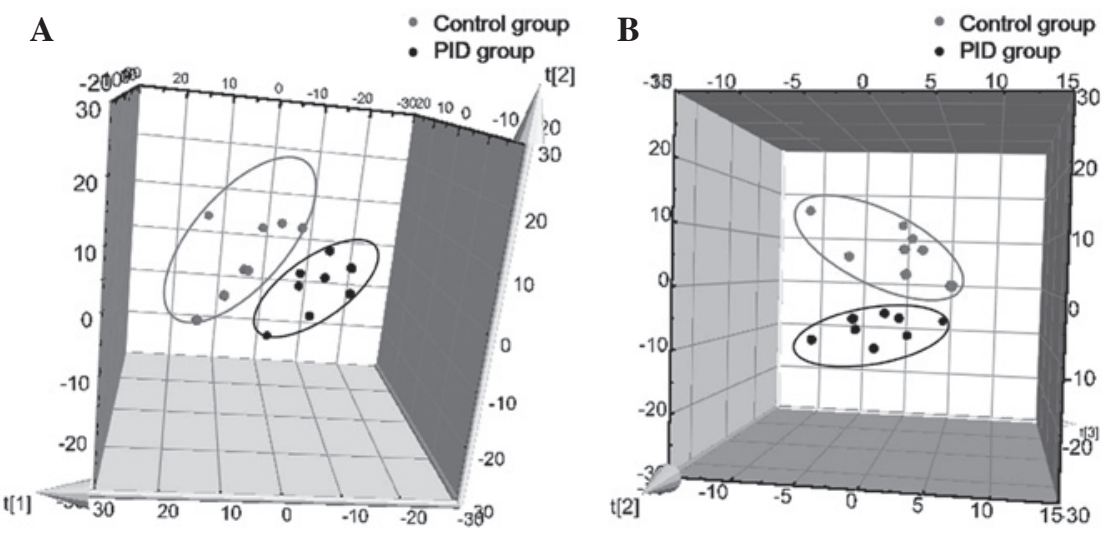

Figure 4. Rats in the PID group exhibited a distinct metabolic profile from the control rats. (A) PCA 3-D scores and (B) PLS-DA 3-D scores plots, based on the metabolomic data obtained from urine. The gray data points indicate control rats, and the black data points indicate PID rats. PID, pelvic inflammatory disease; PCA, principal component analysis; DA, discriminant analysis.

\section{A}

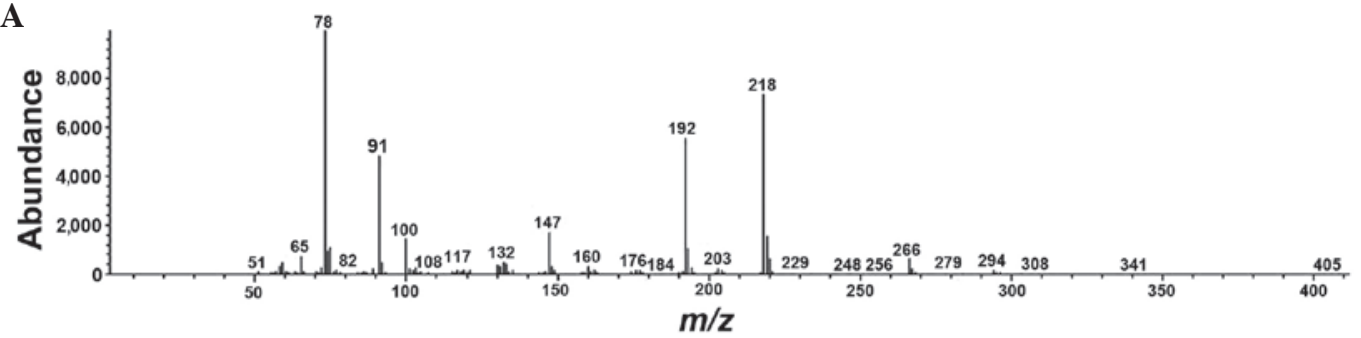

B

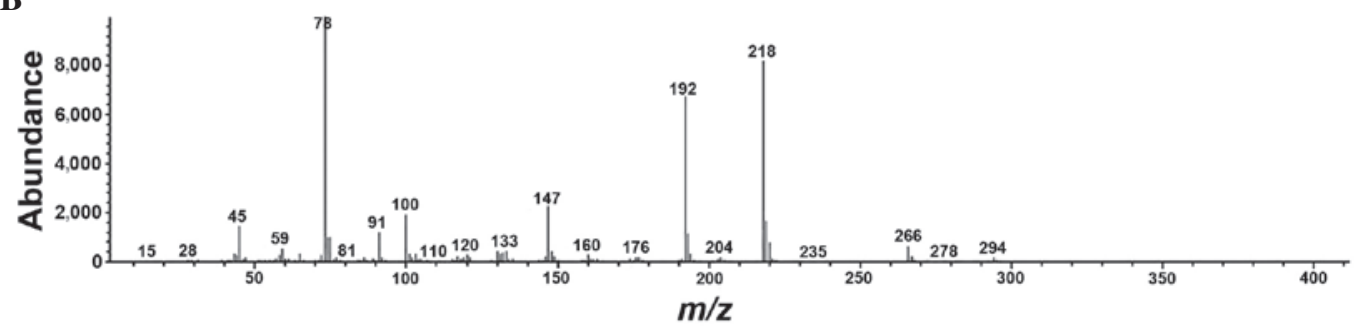

Figure 5. Representative mass spectrum of (A) phenylalanine in rat urine and (B) phenylalanine standard. $\mathrm{m} / \mathrm{z}$, mass to charge ratio.

are four amino acids, three fatty acids, nine organic acids and two sugars. The levels of 14 metabolites were notably increased in the urine of the rats with PID compared with their levels in control rats, but the levels of four metabolites, namely glycine, glucose, galactose and arachidonate, were decreased.

\section{Discussion}

To the best of our knowledge, no previous study has delineated the metabolomic changes that are associated with PID. The aim of the present study was to examine the PID-associated 


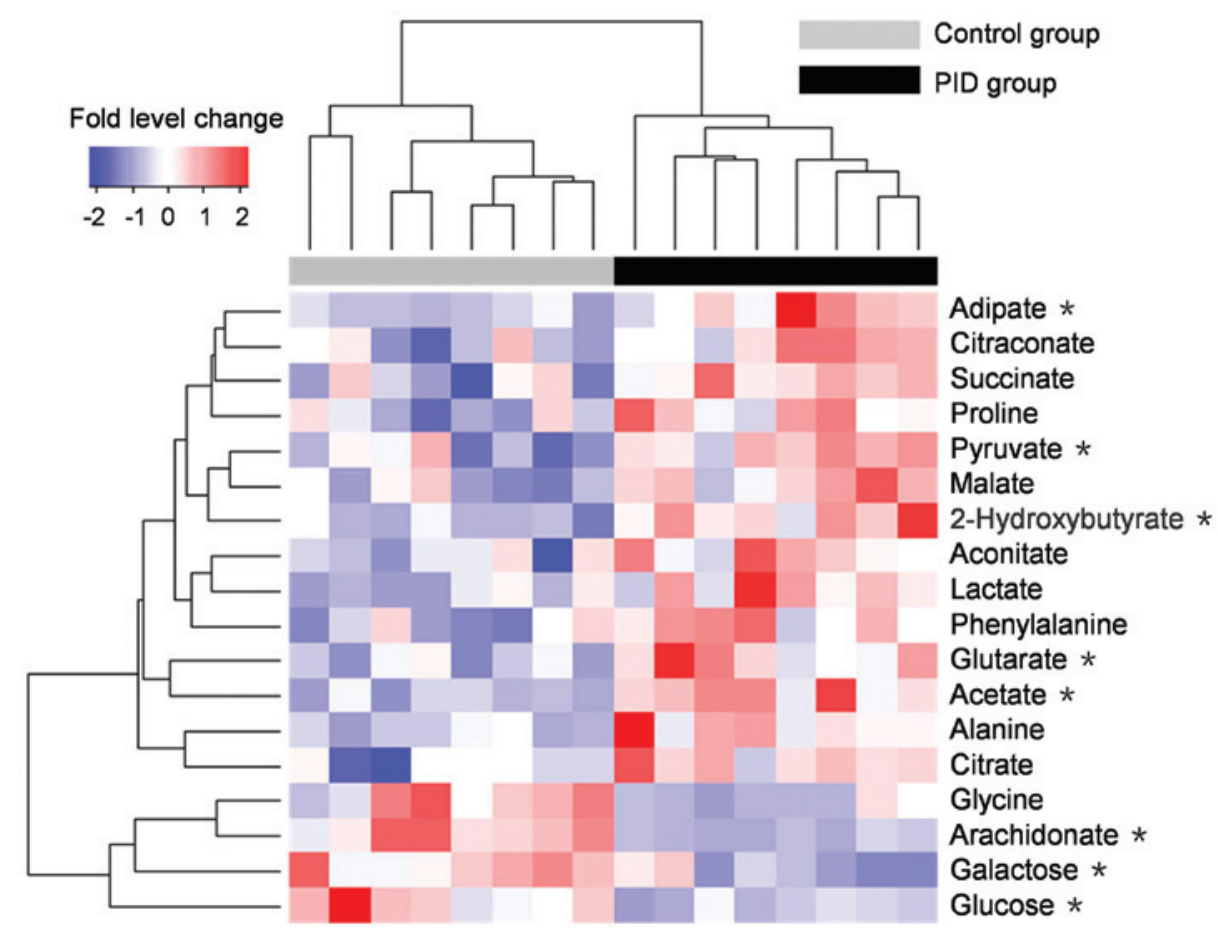

Figure 6. Heat map showing the unsupervised hierarchical clustering of 18 differentiating metabolites across the control and PID groups. The PID group and control group are indicated by black and gray bars, respectively. The columns represent the individual rats, and the rows indicate differentiating metabolites. Increases in the intensities of red and blue indicate elevations and decreases, respectively, in the levels of a metabolite relative to the median metabolite production . $\mathrm{P}<0.01$ vs. the control group, $\mathrm{n}=8$. PID, pelvic inflammatory disease.

disturbance of metabolism in rats with the further aim of identifying potential metabolite biomarkers for use in the diagnosis of this disease.

Toll-like receptors (TLRs) play a key role in evoking the inflammatory response to microbial infection in the genital tract (19). U. urealyticum (20) can be recognized by TLR 2 , and pathogenic E. coli (21) causes an inflammatory response through TLR 4. Furthermore, both U. urealyticum and E. coli are common pathogens of PID (22). Therefore, to activate multiple TLRs and induce a quick and enhanced inflammatory response in the genital tract, a solution containing a mixture of $U$. urealyticum and E. coli was used to construct a PID rat model, as in previous studies $(16,17)$. Following infection, mass neutrophil and lymphocyte infiltrations indicated inflammation in the uterus and fallopian tube (Fig. 1). The elevation of CRP levels in plasma has been used to differentiate inflammatory from non-inflammatory pelvic pathology in vivo (23). CRP examination has been included in the criteria for the diagnosis of PID proposed in the USA (24). In the PID model in the present study, the elevated CRP levels in plasma (Fig. 2A) illustrated the inflammatory response. The pro-inflammatory cytokine IL-1 $\beta$ is a key factor in the pathological development of PID (25), and the cytokine IL-6 is involved in the processes of chronization and suppression of the synthesis of IL- $1 \beta$ in the second phase of the immune response (26). Both IL-1 $\beta$ and IL-6 exhibit significant elevation in patients with PID (27). Hence, as shown in Fig. 2B and C, the elevated expression of IL- $1 \beta$ and IL- 6 in the uterus and fallopian tube validated the local inflammatory response in the rat PID model.

The biochemical reactions associated with the differentiating metabolites can be determined using the Kyoto Encyclopedia of Genes and Genomes (KEGG; http://www. genome.jp/kegg/) and the Human Metabolome Database (HMDB, http://www.hmdb.ca/). The present study illustrated that the comprehensive metabolic profile changes associated with PID in rats are associated with sugar metabolism, the citric acid cycle (TCA cycle), amino acid metabolism and fat acid metabolism. The concurrence of reductions in the levels of sugars (glucose and galactose) and an increase in pyruvate levels indicated enhanced glycolysis in PID rats. In addition, the upregulation of citrate suppresses citrate synthase activity, followed by increased lactate production from pyruvate. The elevated levels of intermediate products in the TCA cycle, including citrate, aconitate, succinate and malate, suggest possible defects in the mitochondrial respiratory system, and the concentration changes of alanine, glycine, phenylalanine and proline indicate alterations of amino acid metabolism. The increase of glutarate and adipate in PID rats illustrates an abnormality of fat acid metabolism that is also present in arthritis $(28,29)$. Leukotriene B4 (LTB4) is able to promote leucocyte recruitment (30), and increased prostaglandin E2 (PGE2) levels lead to edema and a hyperalgesic response (31). The overproduction of both in women with PID (32) may be involved in modulation of the inflammatory response. As arachidonate is the primary precursor of LTB4 and PGE2, high levels of production of LTB4 and PGE2 will consume considerable arachidonate. Consistently, a reduction in the levels of arachidonate was observed in the rats with PID in the present study. The elevation of citraconate also occurs in experimental arthritis (29), indicating a modulation of branched pentadioic acid metabolism relating to an inflammatory response. 2-Hydroxybutyrate is a by-product of ophthalmate synthesis, a high level of which indicates glutathione depletion (33). The increased levels 2-hydroxybutyrate observed in rats with PID in 
this study may suggest a shortage of glutathione and oxidative stress in the genital tract, which is also observed in endometriosis (34). Acetic acid is a substance existing as an antibacterial agent in the vaginal lubrication fluid of humans (35). Notably, elevated pyruvate levels may have promoted acetate production in the present study, as pyruvate can be metabolized by pyruvate dehydrogenase to produce acetate (36). This elevation of acetate levels may be considered as a physiological response to defend against the following infection.

In conclusion, a PID model was constructed by multi-pathogenic infection of the upper genital tract in rats. Infiltration of inflammatory cells and elevated expression levels of cytokines in the uterus and fallopian tube validated the disease model. The results reveal PID-associated metabolomic changes in the rat PID model. Eighteen differentiating metabolites were found, which are associated with sugar metabolism, the TCA cycle, amino acid metabolism and fatty acid metabolism. These metabolites could be potential biomarkers of PID. This study also offers a new approach to evaluate the effect of anti-PID drugs in pre-clinical or clinical trials.

\section{Acknowledgements}

The authors thank the Clinical Laboratory Department and Department of Pathology in the Maternal and Child Health Hospital of Hunan Province for experimental support. The present study was supported by the National Natural Science Foundation of China (grant no. 81501218), China Postdoctoral Science Foundation (grant no. 2015M582330), Science and Technology Project of Hunan Province (grant no. 2015RS4054) and Administration of Traditional Chinese Medicine of Hunan Province (grant no. 201402).

\section{References}

1. World Health Organization: Global incidence and prevalence of selected curable sexually transmitted infections: 2008. Reprod Health Matters 20: 207-209, 2012.

2. Quentin R and Verdon R: Microbiologic basis of diagnosis and treatment of pelvic inflammatory disease. J Gynecol Obstet Biol Reprod (Paris) 41: 850-863, 2012 (In French).

3. Saini S, Gupta N, Aparna, Batra G and Arora DR: Role of anaerobes in acute pelvic inflammatory disease. Indian J Med Microbiol 21: 189-192, 2003.

4. Zhang DZ, Wen JY, Zhou WC and Wu XY: Pathogenic bacteria distribution and drug resistance isolated from women with pelvic inflammatory disease. Zhonghua Yi Yuan Gan Ran Xue Za Zhi 19: 1747-1750, 2009 (In Chinese).

5. Soper DE: Pelvic inflammatory disease. Obstet Gynecol 116: 419-428, 2010.

6. Blenning CE, Muench J, Judkins DZ and Roberts KT: Clinical inquiries. Which tests are most useful for diagnosing PID? J Fam Pract 56: 216-220, 2007.

7. Jaiyeoba $\mathrm{O}$ and Soper DE: A practical approach to the diagnosis of pelvic inflammatory disease. Infect Dis Obstet Gynecol 2011: 753037, 2011.

8. Nicholson JK, Lindon JC and Holmes E: 'Metabonomics': Understanding the metabolic responses of living systems to pathophysiological stimuli via multivariate statistical analysis of biological NMR spectroscopic data. Xenobiotica 29: 1181-1189, 1999.

9. Coen M, Holmes E, Lindon JC and Nicholson JK: NMR-based metabolic profiling and metabonomic approaches to problems in molecular toxicology. Chem Res Toxicol 21: 9-27, 2008.

10. Asiago VM, Alvarado LZ, Shanaiah N, Gowda GA, Owusu-Sarfo K, Ballas RA and Raftery D: Early detection of recurrent breast cancer using metabolite profiling. Cancer Res 70: 8309-8318, 2010.
11. Sreekumar A, Poisson LM, Rajendiran TM, Khan AP, Cao Q, Yu J, Laxman B, Mehra R, Lonigro RJ, Li Y, et al: Metabolomic profiles delineate potential role for sarcosine in prostate cancer progression. Nature 457: 910-914, 2009.

12. Sato Y, Suzuki I, Nakamura T, Bernier F, Aoshima K and Oda Y. Identification of a new plasma biomarker of Alzheimer's disease using metabolomics technology. J Lipid Res 53: 567-576, 2012.

13. Fiehn O, Kopka J, Dormann P, Altmann T, Trethewey RN and Willmitzer L: Metabolite profiling for plant functional genomics. Nat Biotechnol 18: 1157-1161, 2000.

14. Wiklund S, Johansson E, Sjöström L, Mellerowicz EJ, Edlund U, Shockcor JP, Gottfries J, Moritz T and Trygg J: Visualization of GC/TOF-MS-based metabolomics data for identification of biochemically interesting compounds using OPLS class models. Anal Chem 80: 115-122, 2008.

15. Hong Z, Lin Z, Liu Y, Tan G, Lou Z, Zhu Z, Chai Y, Fan G, Zhang $J$ and Zhang L: Innovative microwave-assisted oximation and silylation procedures for metabolomic analysis of plasma samples using gas chromatography-mass spectrometry. J Chromatogr A 1254: 14-22, 2012.

16. Cai XF, Chen LF, Wang ZL and Gu YE: Effect of acupoint injection by Astragalus injection on local SIgA and pathomorphology changes in rats with chronic pelvic inflammatory disease. Zhongguo Zhong Yao Za Zhi 31: 1361-1364, 2006 (In Chinese).

17. Li X, Guo J, Shi Z and Nie J: Effect of Fuke Qianjin tablets on inflammatory cytokines in blood serum in rats with chronic pelvic inflammatory disease. Zhongguo Shi Yan Fang Ji Xue Za Zhi 19: 226-228, 2013 (In Chinese).

18. Benjamini Y and Hochberg Y: Controlling the false discovery rate: A practical and powerful approach to multiple testing. J R Stat Soc Series B Stat Methodol 57: 289-300, 1995.

19. Sonnex C: Toll-like receptors and genital tract infection. Int J STD AIDS 21: 153-157, 2010

20. He J, You X, Zeng Y, Yu M, Zuo L and Wu Y: Mycoplasma genitalium-derived lipid-associated membrane proteins activate NF-kappaB through toll-like receptors 1,2 and 6 and CD14 in a MyD88-dependent pathway. Clin Vaccine Immunol 16: 1750-1757, 2009.

21. Sheldon IM, Rycroft AN, Dogan B, Craven M, Bromfield JJ, Chandler A, Roberts MH, Price SB, Gilbert RO and Simpson KW: Specific strains of Escherichia coli are pathogenic for the endometrium of cattle and cause pelvic inflammatory disease in cattle and mice. PLoS One 5: e9192, 2010.

22. Zhou B, Cong L and Sha Y: Pathogens of transmitted disease in the pathogenesis of acute pelvic inflammatory disease. Zhonghua Fu Chan Ke Za Zhi 36: 539-541, 2001 (In Chinese).

23. Angerman NS, Evans MI, Moravec WD, Schumacher GF and Hajj SN: C-reactive protein in the evaluation of antibiotic therapy for pelvic infection. J Reprod Med 25: 63-66, 1980.

24. Workowski KA and Berman S; Centers for Disease Control and Prevention (CDC): Sexually transmitted diseases treatment guidelines, 2010. MMWR Recomm Rep 59: 1-110, 2010.

25. Cheng W, Shivshankar P, Li Z, Chen L, Yeh IT and Zhong G: Caspase-1 contributes to Chlamydia trachomatis-induced upper urogenital tract inflammatory pathologies without affecting the course of infection. Infect Immun 76: 515-522, 2008.

26. Trunov A, Obukhova O, Gorbenko O, Shvayk A and Trunova L: Cytokines, estradiol and progesterone in the plasma of women of reproductive age with pelvic inflammatory disease in remission. Adv Biosci Biotechnol 4: 727, 2013.

27. Lee SA, Tsai HT, Ou HC, Han CP, Tee YT, Chen YC, Wu MT, Chou MC, Wang PH and Yang SF: Plasma interleukin-1beta, $-6,-8$ and tumor necrosis factor-alpha as highly informative markers of pelvic inflammatory disease. Clin Chem Lab Med 46: 997-1003, 2008.

28. Weljie AM, Dowlatabadi R, Miller BJ, Vogel HJ and Jirik FR: An inflammatory arthritis-associated metabolite biomarker pattern revealed by $1 \mathrm{H}$ NMR spectroscopy. J Proteome Res 6: 3456-3464, 2007.

29. Yue R, Zhao L, Hu Y, Jiang P, Wang S, Xiang L, Liu W, Zhang W and Liu R: Rapid-resolution liquid chromatography TOF-MS for urine metabolomic analysis of collagen-induced arthritis in rats and its applications. J Ethnopharmacol 145: 465-475, 2013.

30. Hotter G, Closa D, Prats N, Pi F, Gelpí E and Roselló-Catafau J: Free radical enhancement promotes leucocyte recruitment through a PAF and LTB4 dependent mechanism. Free Radic Biol Med 22: 947-954, 1997. 
31. Seibert K, Zhang Y, Leahy K, Hauser S, Masferrer J, Perkins W, Lee $L$ and Isakson P: Pharmacological and biochemical demonstration of the role of cyclooxygenase 2 in inflammation and pain. Proc Natl Acad Sci USA 91: 12013-12017, 1994.

32. Heinonen PK, Aine R and Seppälä E: Peritoneal fluid leukotriene B4 and prostaglandin E2 in acute salpingitis. Gynecol Obstet Invest 29: 292-295, 1990.

33. Soga T, Baran R, Suematsu M, Ueno Y, Ikeda S, Sakurakawa T, Kakazu Y, Ishikawa T, Robert M, Nishioka T and Tomita M: Differential metabolomics reveals ophthalmic acid as an oxidative stress biomarker indicating hepatic glutathione consumption. J Biol Chem 281: 16768-16776, 2006.
34. Jana SK, Dutta M, Joshi M, Srivastava S, Chakravarty B and Chaudhury K: ${ }^{1} \mathrm{H}$ NMR based targeted metabolite profiling for understanding the complex relationship connecting oxidative stress with endometriosis. Biomed Res Int 2013: 329058, 2013.

35. Valore EV, Park CH, Igreti SL and Ganz T: Antimicrobial components of vaginal fluid. Am J Obstet Gynecol 187: 561-568, 2002.

36. Krebs HA and Johnson WA: Metabolism of ketonic acids in animal tissues. Biochem J 31: 645-660, 1937. 